\title{
High Pressure Cell for Small-Angle Neutron and Light Scattering Studies of Phase Transitions in Complex Liquids
}

\author{
Hiroyuki Takeno, Michihiro Nagao, ${ }^{*}$ Yoshiaki Nakayama, ${ }^{* *}$ Hirokazu Hasegawa, \\ Takeji Hashimoto, ${ }^{\dagger}$ Hideki Seto, ${ }^{*}$ and Masayuki ImaI ${ }^{* * *}$ \\ Department of Polymer Chemistry, Graduate School of Engineering, Kyoto University, Kyoto 606-01, Japan \\ * Faculty of Integrated Arts and Sciences, Hiroshima University, Higashi-Hiroshima 739, Japan \\ **Asahi Chemical Industry Co., Ltd., Nobeoka, Miyazaki 882, Japan \\ *** Institute for Solid State Physics, The University of Tokyo, Ibaraki 319-11, Japan
}

(Received April 14, 1997)

\begin{abstract}
High pressure cells for both small-angle neutron scattering and light scattering studies have been designed and constructed to investigate phase transitions of complex liquids. The cells can be used for polymer blends, polymer solutions, liquid mixtures of small-molecules, etc. Pressure up to $200 \mathrm{MPa}$ can be applied to the cells through a pressurizing liquid. The sample is separated from the pressurizing liquid by a free piston or an O-ring. In addition, time-resolved measurements of phase transition processes induced by a pressure drop can be carried out using the cells. The pressure is stable within $0.5 \mathrm{MPa}$ for the duration of several hours. Temperature can be controlled between 273 and $523 \mathrm{~K}$ with an accuracy of $\pm 0.005 \mathrm{~K}$ during the measurement time of 30 minutes. In this paper, we show two preliminary experimental results as examples of applications of the cells: (i) a pressure effect on the miscibility and order-disorder transition temperature of a diblock copolymer and its microphase separation process by a pressure jump, and (ii) a pressure induced critical phenomenon in a three-component microemulsion system.

KEY WORDS High Pressure Cell / Small-Angle Neutron Scattering / Light Scattering / Complex Liquids / Polymer / Microemulsion /
\end{abstract}

Recently, numerous studies have been made on phase transitions of complex liquids in various research fields. $^{1,2}$ Small-angle neutron scattering (SANS) and light scattering (LS) have made a great contribution to phase transition studies of critical phenomena, miscibility and phase separation processes, etc. Temperature has so far been used as the thermodynamic variable in most of the phase transition studies. However, it has been pointed out that pressure is also an important thermodynamic parameter in phase transitions of complex liquids. ${ }^{3-9}$ Pressure affects the free volumes of complex liquids and hence their phase transitions. Especially, in a polymer system, the combinatorial entropy term in Flory-Huggins expression of free energy of mixing is inversely proportional to the degree of polymerization $N .{ }^{10}$ It means that the contribution of the other factors to the free energy of mixing becomes $N$ times larger than that in a small molecular system, and therefore, it is expected that the free volume effects also become $N$ times larger.

With these points in mind, we have designed and constructed two high pressure cells, one for SANS and the other for LS measurements. By using the cells, we can investigate the effect of pressure on the miscibility and the critical phenomena of complex liquids. In addition, we can study the kinetics and the phase transition process induced by a pressure jump. So far, many experimental attempts have been made to study the dynamics of phase transitions of complex liquids by temperature jumps. However, a temperature jump cannot be completed instantaneously and it takes some time for the target temperature to be attained by thermal conduction. Unless the time is sufficiently short relative to the rate of the phase transition, it is extremely difficult to investigate the dynamics. The response of pressure in a pressure jump is much faster and enables us to follow the dynamics of a rapid phase transition. As an example of dynamic measurements by a pressure jump, we show a time-resolved neutron scattering measurement of the microphase separation process of a diblock copolymer.

Several high pressure cells for the scattering studies of complex liquids have been reported to date. Highpressure cells for SANS were reported by the groups in Jülich $^{5,6}$ and at National Institute of Standards and Technology (NIST), ${ }^{7}$ for small-angle X-ray scattering (SAXS) by the group in Princeton University ${ }^{8}$ and for LS from supercritical liquids and solutions by Kojima et al. ${ }^{9}$ Comparisons of the maximum applicable pressure $P_{\max }$, the stability of pressure $\Delta P$, the measurable temperature range $T_{\text {range }}$ and the stability of temperature $\Delta T$ for their cells are summarized in Table I. The maximum applicable pressure is 200 [MPa] for Jülich, 100 [MPa] for NIST, 300 [MPa] for Princeton, 34.3 [MPa] for Kojima et al. and 200 [MPa] for our cell. $\Delta P$ are of the order of $0.5 \%$ of the applied pressure for Princeton, 0.01 [MPa] for Kojima et al. and 0.5 [MPa] for the duration of several hours for our cell. Temperature can be varied from 253 to $473 \mathrm{~K}$ with accuracy of $\pm 0.1 \mathrm{~K}$ for Jülich, from 273 to $458 \mathrm{~K}$ with accuracy of $\pm 0.05 \mathrm{~K}$ for Princeton, to $573 \mathrm{~K}$ with accuracy of $\pm 0.1 \mathrm{~K}$ for Kojima et al. and from 273 to $523 \mathrm{~K}$ with accuracy of $\pm 0.005 \mathrm{~K}$ during the 30 minutes measurement for our cell. Thus, our cell has equal or higher performance compared with other high pressure cells.

Among many features of these high-pressure cells, we are most interested in the methods employed to separate the sample and the pressurizing liquid. The sample is separated from the pressurizing liquid by an O-ring in

† To whom correspondence should be addressed. (Tel: (+81)-75-753-5604; fax: (+81)-75-753-4864; e-mail: hashimoto@alloy.polym.kyoto-u.ac.jp) 
Table I.

\begin{tabular}{lccccc}
\hline & Jülich & NIST & Princeton & $\begin{array}{c}\text { Kojima } \\
\text { et al. }\end{array}$ & This work \\
\hline$P_{\text {max }} / \mathrm{MPa}$ & 200 & 100 & 300 & 34.3 & 200 \\
$\Delta P / \mathrm{MPa}$ & - & - & $0.5 \%^{\mathrm{a}}$ & 0.01 & 0.5 \\
$T_{\text {range }} / \mathrm{K}$ & $253-473$ & - & $273-458$ & -573 & $273-523$ \\
$\Delta T / \mathrm{K}$ & \pm 0.1 & - & \pm 0.05 & \pm 0.1 & \pm 0.005 \\
\hline
\end{tabular}

${ }^{\mathrm{a}}$ The accuracy and resolution of the sensor are of the order of $0.5 \%$ of the applied pressure.

the two cells for SANS in Jülich and at NIST. The pressurizing liquid comes into direct contact with sample in the cell of the Princeton group. Kojima et al. used the sample itself (supercritical polymer solutions) as the pressurizing liquid.

On the other hand, we employ a free piston and an O-ring, and use either of them to separate the liquid sample from the pressurizing liquid depending on the viscosity of the sample. It seems that these methods are most reliable for the separation. The free piston is used for low viscosity liquids such as dilute polymer solutions or small-molecular liquids and the O-ring is used for high viscosity liquids such as polymer blends or block copolymers (these details are discussed in INSTRUMENTATION section). It is difficult to keep low viscosity liquids inside the O-ring. By using both methods properly, not only high viscosity liquids but also low viscosity liquids can be measured in our cells.

Moreover, we have designed the body of our cells with two screw holes besides that for applying pressure through a pressurizing liquid, so that we can make various modifications, e.g., (i) temperature and pressure of the sample can be directly measured by inserting a pressure gauge and a thermometer into these holes, (ii) an apparatus for pressure drop to an arbitrary pressure can be fixed on the cells by using these holes, (iii) an apparatus for pressure release can be fixed on the cells to avoid the drift of pressure with an increase of temperature as pointed out by NIST group, ${ }^{7}$ etc. The additional advantage of our cells is that time-resolved measurements after pressure jumps can be performed. In addition, by using both cells for SANS and LS, we can investigate structures (fluctuations) of a very wide spatial scale.

In this paper, we report on the design and the construction of the high-pressure cells for SANS and LS studies of complex liquids and also on two preliminary SANS experiments with the cell: (i) pressure effects on the miscibility and the order-disorder transition temperature $\left(T_{\text {ODT }}\right)$ of a deuterated polybutadiene-blockpolyisoprene diblock copolymer (DPB-HPI) and its microphase separation process by a pressure jump, (ii) a pressure-induced critical phenomenon in an AOT (dioctyl sulfosuccinate sodium salt) $/ \mathrm{D}_{2} \mathrm{O} / n$-decane threecomponent microemulsion system. We show that pressure strongly affects the SANS profiles of these systems.

\section{INSTRUMENTATION}

\section{SANS and LS Cells}

The sample is separated from the pressurizing liquid in two ways: by a free piston $\{1\}$ (Figure 1a) and by an
O-ring made of silicon $\{2\}$ (Figure 1b). These figures show the cross-sectional views of the high pressure cell for SANS studies of complex liquids (hereafter shortened to "the SANS cell") in a plane parallel to the incident beam, while Figure 1c shows that perpendicular to the incident beam viewed from the detector side. Figures $2 \mathrm{a}$ and $2 \mathrm{~b}$ show cross-sectional views of the high pressure cell for LS studies ("the LS cell") in a plane parallel to and perpendicular to the incident beam viewed from the detector side, respectively. The only difference between the SANS and LS cells is the aperture angles of the cell windows both on the incident and scattered sides.

As shown in Figures $1 \mathrm{a}$ and $1 \mathrm{~b}$, the free piston $\{1\}$ is placed inside the stainless pipe $\{3\}$ which is screwed into the body of the cell $\{6\}$ and the O-ring $\{2\}$ is placed between the sapphire windows $\{4\} .20 \mathrm{~mm}$ thick sapphire windows of a truncated-cone shape $\{4\}$ are attached to a frame made of stainless-steel (SUS630) $\{5\}$ and the sample is sandwiched between the bottom sides of the windows. The sapphire windows are lapped to fit the stainless-steel frame. As tapered windows are used, they are tightly fixed on the frame as pressure is applied and can hold pressure up to 200 [MPa]. The top and bottom diameters of the truncated-cone windows are $21 \mathrm{~mm}$ and $30 \mathrm{~mm}$, respectively. The body of the cell $\{6\}$ is made of stainless-steel which is surrounded by a cast-aluminum heater $\{7\}$. Besides the silicon O-ring $\{2\}$, three O-rings, two made of copper $\{8\}$ and one made of Teflon containing copper powder $\{9\}$, are the pressure seals between the body of the cell $\{6\}$ and the stainless-steel frames $\{5\}$ fixed on the sapphire windows. The O-ring made of Teflon containing copper powder $\{9\}$ is placed between the two copper O-rings $\{8\}$. A stainless-steel screw $\{10\}$ which has an opening for the neutron beam path in the center is tightened on the body of the cell in order to fix the windows. The opening of the screw of the LS cell is much smaller than that of the SANS cell as shown in Figures 1a and 2a. As shown in Figure 1, the SANS cell has a tapered opening on the detector side. Maximum and minimum diameters of the opening are 16 and $10 \mathrm{~mm}$, respectively. A $q$-range less than $2.0 \times 10^{-3}\left[\AA^{-1}\right]$ for $\lambda=7[\AA]$ can be covered with the SANS cell, where $q$ is a scattering vector and defined as $q=(4 \pi / \lambda) \sin (\theta / 2)$ with $\lambda$ and $\theta$ being the wavelength in the medium and the scattering angle, respectively.

The LS cell has a slit opening on the detector side (Figure 2). The opening is tapered at an angle of $45^{\circ}$ in one direction in order to cover a wide $q$ range. The range of scattering angles in the sample is within $28^{\circ}$, which means that the $q$ range is within $7.23 \times 10^{-4}\left[\AA^{-1}\right]$ for $\lambda=6328[\AA]$. Sample thickness can be adjusted by a metal spacer $\{11\}$ between 0.2 and $3 \mathrm{~mm}$.

The body of the cell has three screw holes A, B, and $\mathrm{C}$ (Figures $1 \mathrm{c}$ and $2 \mathrm{~b}$ ). One of them (A) is for applying pressure through a pressurizing liquid. By inserting a thermometer $\{12\}$ or a pressure gauge $\{12\}$ into the other holes (B and C), we can directly measure the temperature and the pressure of the sample. When they are not used, the screw holes $\{12\}$ are plugged with stoppers.

\section{Temperature and Pressure Control Units}

A schematic diagram of the high pressure apparatus including the pressure gauge and the temperature con- 


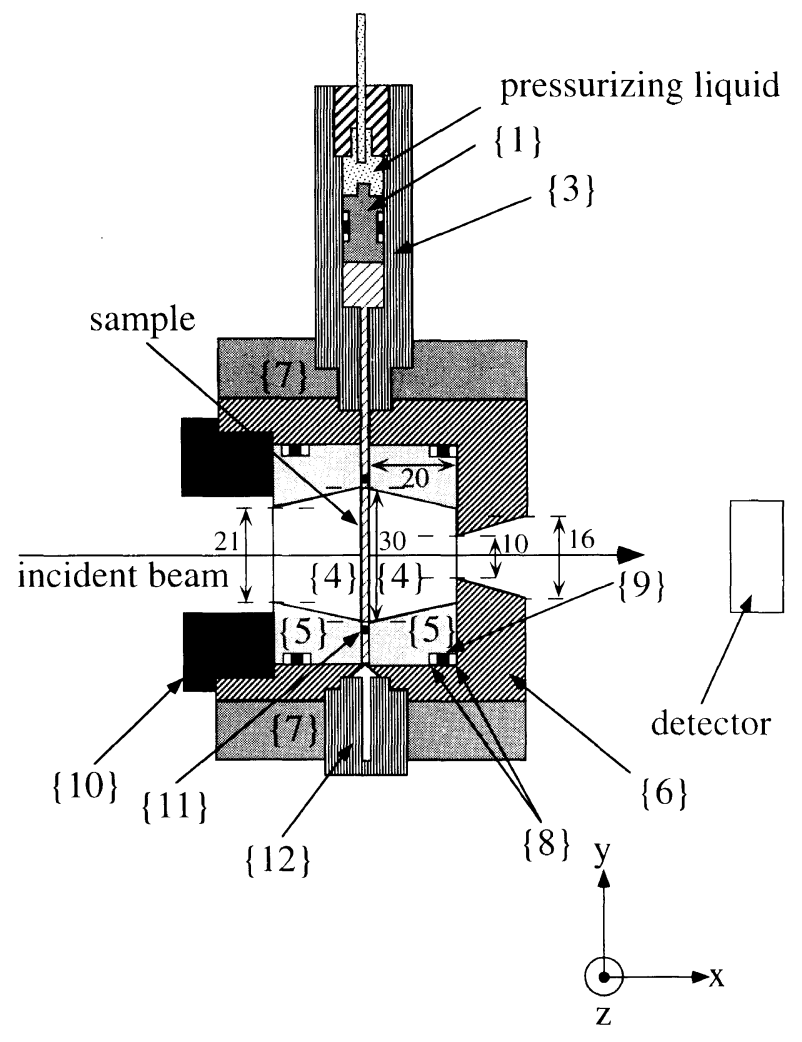

(a)

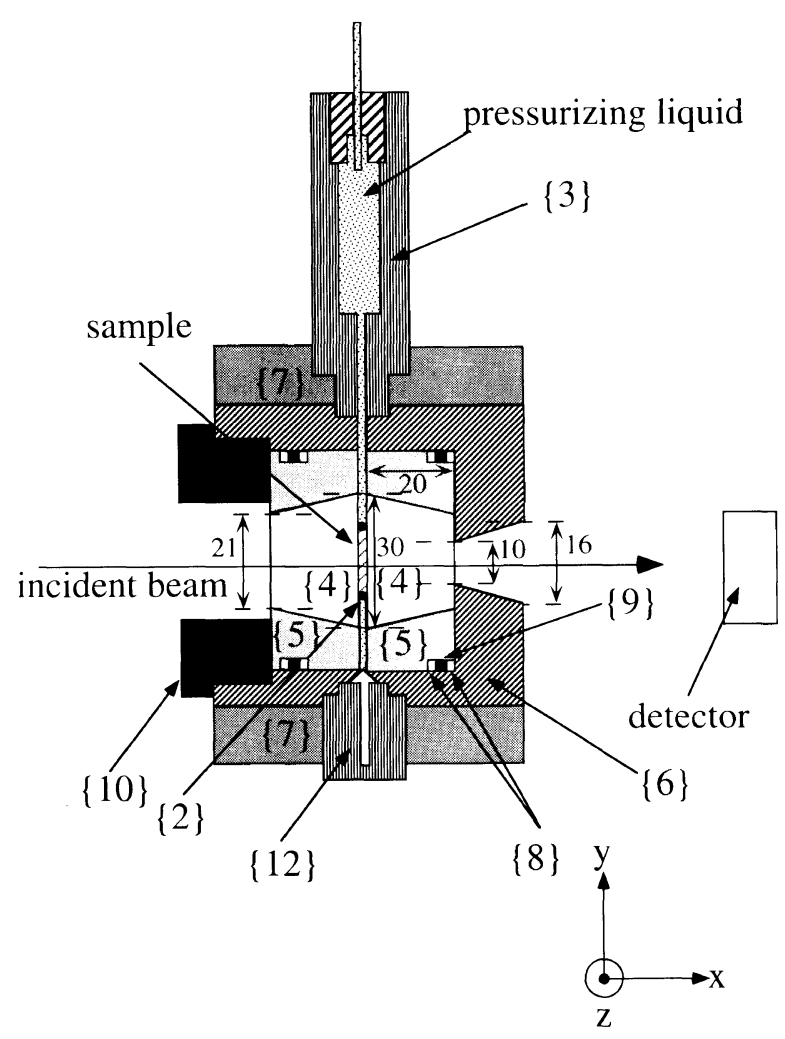

(b)

troller is shown in Figure 3. The body of the cell is connected by a flexible stainless-steel capillary tube $\{1\}$ to a manually operated pump $\{2\}$ filled with a pressurizing liquid such as diethylene glycol or paraffin oil (Figure 3). Pressures up to 200 [MPa] can be generated by the pump which is equipped with a valve for pres-

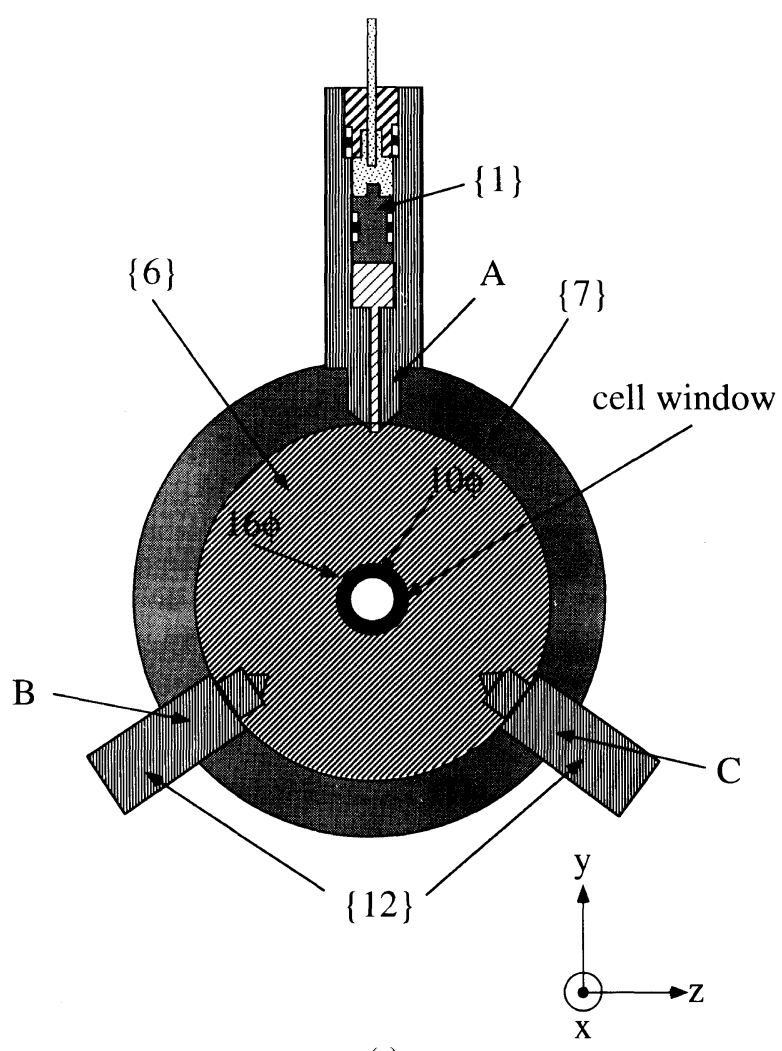

(c)

Figure 1. Cross-sectional views of the high pressure cell for SANS parallel to incident beam: free piston method (a) and O-ring method (b). Cross-sectional views for SANS (c) perpendicular to incident beam. The incident beam is in the $x$ direction from left to right in (a) and (b), and out of the paper in (c). $\{1\}$ : free piston, $\{2\}$ : Silicon O-ring, $\{3\}$ : stainless pipe screwed into the body of the cell, $\{4\}$ : sapphire windows, $\{5\}$ : stainless-steel frames on the sapphire windows, $\{6\}$ : body of the cell made of stainless-steel, $\{7\}$ : cast aluminum heater, $\{8\}$ : copper O-rings, $\{9\}$ : Teflon O-ring containing copper powder, $\{10\}$ : stainless-steel screw which has an opening for the neutron beam in the center, $\{11\}$ : metal spacer, $\{12\}$ : stoppers or a thermometer or a pressure gauge. $\{\mathrm{A}, \mathrm{B}$, and $\mathrm{C}\}$ : screw holes.

sure release $\{3\}$ (Figure 3 ). By opening the valve and releasing the pressure, we can perform dynamic measurements of phenomena induced by the pressure drop. (The current apparatus allows only a pressure drop to 0.1 $[\mathrm{MPa}]$. However, a small modification will allow a pressure drop to an arbitrary pressure.) Pressure is measured with a 6 inch Heise gauge $\{4\}$ (Figure 3 ) which can read up to $250[\mathrm{MPa}]$. The pressure is stable within $\pm 0.5 \mathrm{MPa}$ during an experiment of several hours. The cell can be heated up by a cast aluminum heater $\{7\}$ which encloses the body of the cell. Moreover, the cell is covered with a thermal shield $\{5\}$ (Figure 3), with quartz windows. Temperature is monitored with a platinum resistance $\{6\}$ attached to the body of the cell and controlled by a computer, and another platinum resistance $\{7\}$ is attached to the cast aluminum heater for temperature control. The attainable temperature range is between 273 and $523 \mathrm{~K}$. The accuracy of temperature control is within $\pm 0.005 \mathrm{~K}$ during a measurement time of 30 minutes.

\section{Correction for the Change of Sample Thickness}

There is a structural problem with the cell. As the cells do not have the mechanism to hold the two pieces of the 


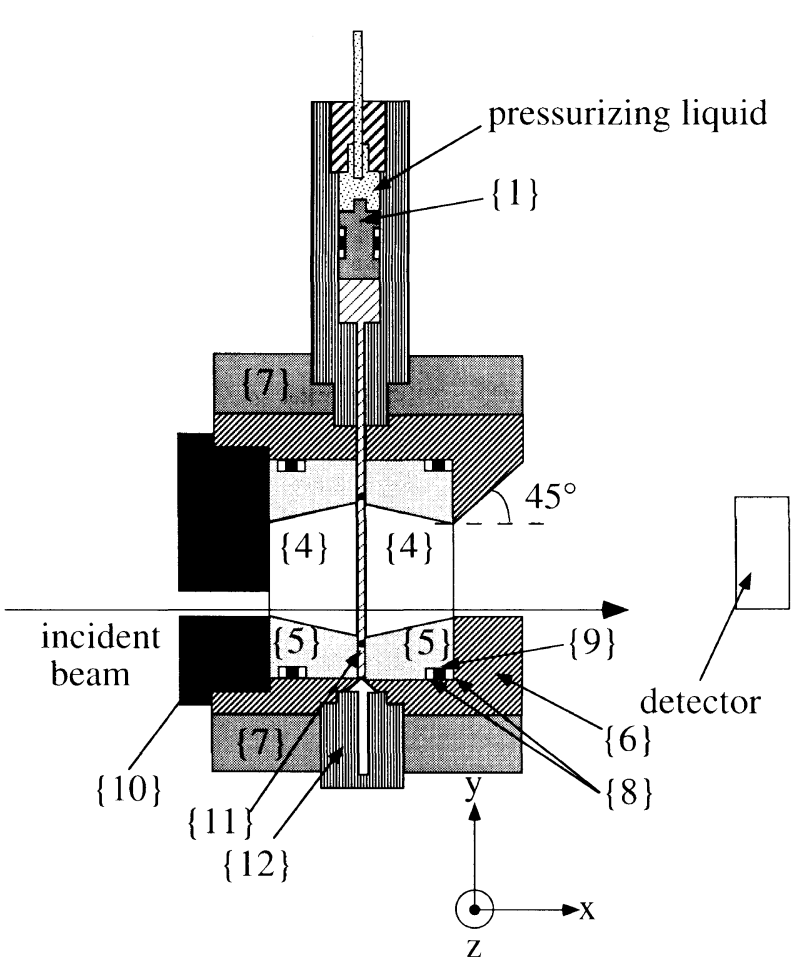

(a)

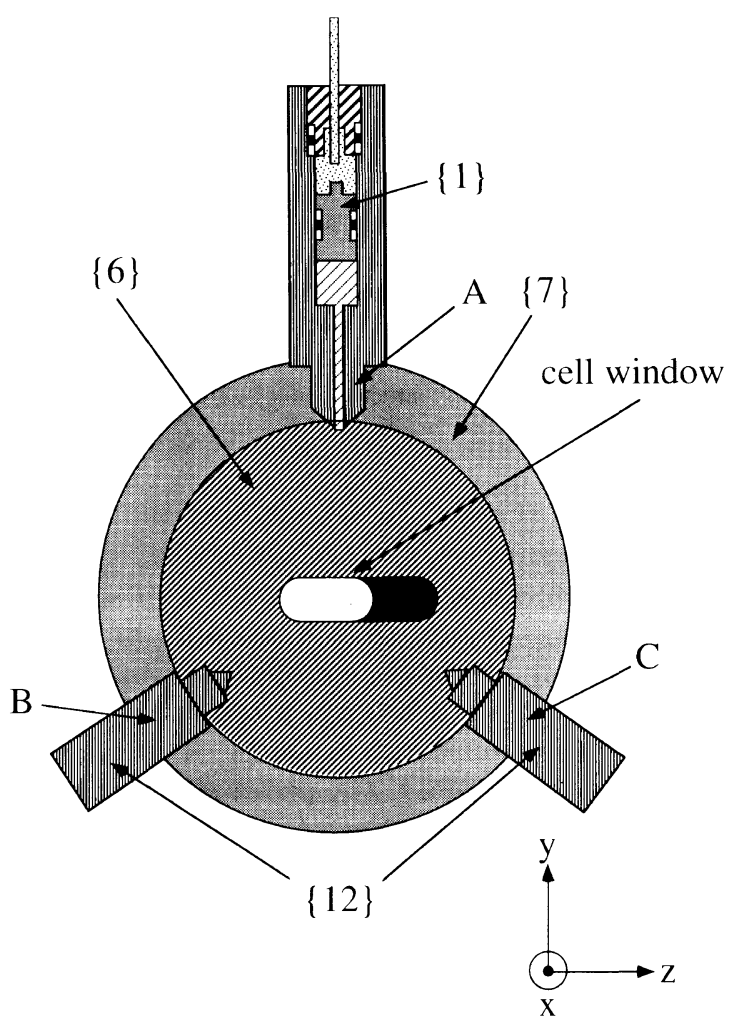

(b)

Figure 2. Cross-sectional views of the high pressure cell for LS parallel (a) and perpendicular (b) to incident beam. The numbers designating the parts of the cell and the direction of incident beam are the same as those in Figure 1.

windows $(\{4\}$ in Figures 1 and 2$)$ in position, they tend to be pushed away from each other as the pressure increases, resulting in an increase in the sample thickness. Therefore, a correction for the change of sample thickness

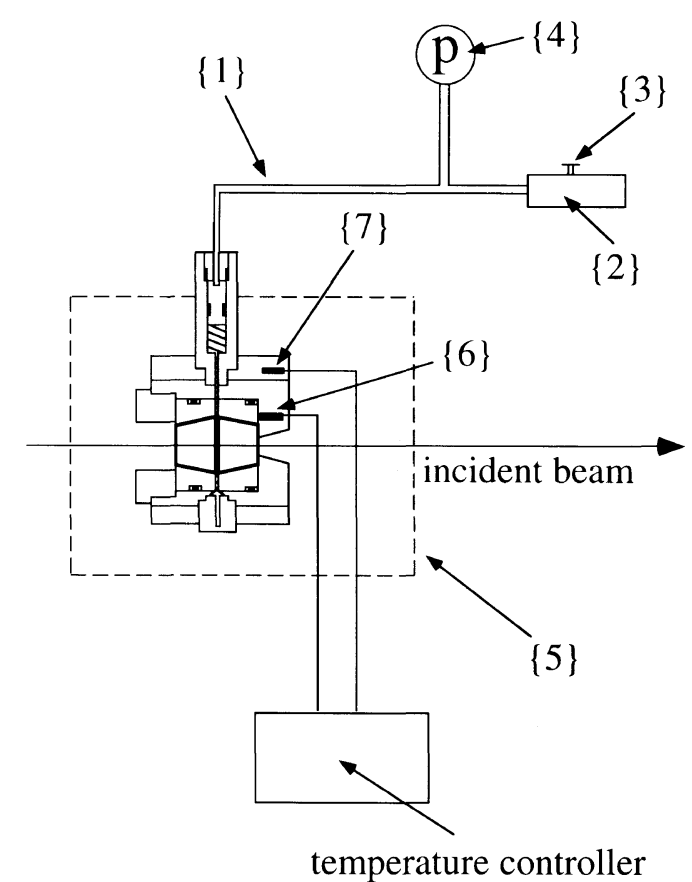

Figure 3. Schematic diagram of the high pressure apparatus. $\{1\}$ : capillary tube, $\{2\}$ : manually-operated pump, $\{3\}$ : valve attached to the pump, $\{4\}$ : Heise pressure gauge, $\{5\}$ : thermal shield, $\{6\}$ : platinum resistor attached to the stainless of the body, $\{7\}$ : platinum resistor attached to cast aluminum heater

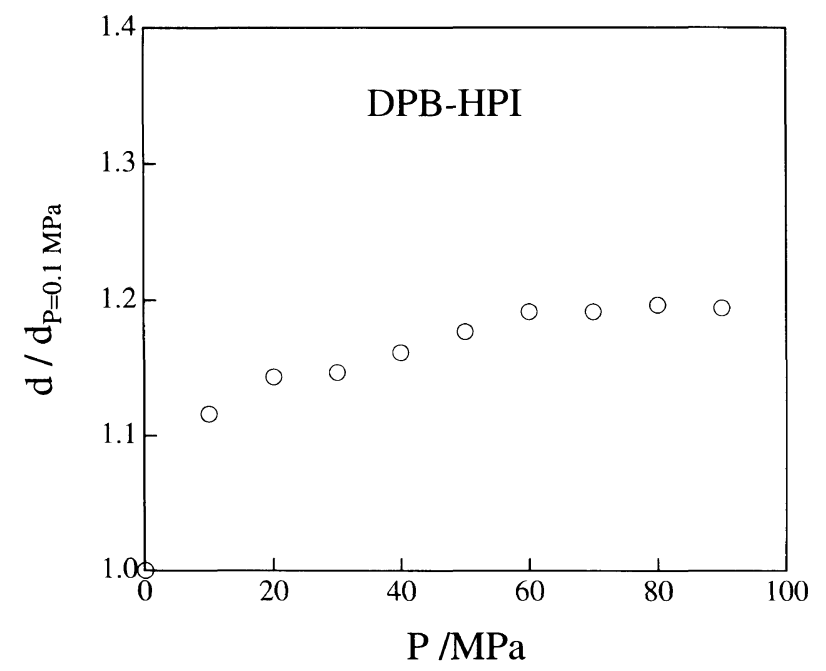

Figure 4. Sample thickness $d$ normalized to that at $P=0.1 \mathrm{MPa}$ plotted as a function of pressure.

with pressure is necessary. The change of sample thickness is estimated by measuring the transmission of polyisoprene (HPI) homopolymer as a function of pressure.

According to Lambert's law, transmission $T_{\mathrm{r}}\left(=I / I_{0}\right)$ of a sample is described by the following expression

$$
T_{\mathrm{r}}=\exp (-\tau d)
$$

where $I$ and $I_{0}$ are the transmitted intensity with and without the sample, respectively, $d$ is the sample thickness, and $\tau$ is the turbidity which is a function of pressure. The value of $\tau$ at $P=0.1[\mathrm{MPa}]$ is obtained by measuring the transmission of HPI homopolymer sample whose thickness is known. The pressure change of the density of HPI homopolymer is estimated from the pressurevolume-temperature (PVT) data in the literature. ${ }^{11}$ 
Assuming the linear relation between the pressure change of $\tau$ and that of the density, the change of the sample thickness is evaluated by using eq 1. Figure 4 shows the pressure dependence of the sample thickness normalized to the thickness at $P=0.1[\mathrm{MPa}]$, which is used for the absolute intensity correction.

\section{EXPERIMENTAL APPLICATIONS: RESULTS AND DISCUSSION}

In this section, we report on preliminary SANS experiments for two systems: (i) deuterated polybutadiene-block-polyisoprene diblock copolymer (DPB-HPI) and (ii) an oil-rich mixture of AOT (dioctyl sulfosuccinate sodium salt), $\mathrm{D}_{2} \mathrm{O}$ and $n$-decane three-component microemulsion. The former experiment was carried out using the SANS cell with the O-ring method (Figure 1b), while the latter with the SANS cell with the free-piston method (Figure 1a). The experimental methods, results and discussion for the former are mentioned in sections Sample Preparation and Characterization of DPB-HPI and Results and Discussion for $D P B-H P I$, respectively, while those for the latter are reported in sections Experiments on a Microemulsion System and Results and Discussion on Microemulsion System.

\section{SANS}

Small-angle neutron scattering (SANS) measurements were performed by using the SANS instrument (SANSU) of the Institute for Solid State Physics of the University of Tokyo at JRR-3M reactor at Japan Atomic Energy Research Institute in Tokai. A neutron beam with the wavelength $\lambda=7[\AA]$ and $\Delta \lambda / \lambda \cong 0.1$ monochromatized by a velocity selector was used. The scattering intensity data were collected by a two dimensional detector and then circularly averaged in order to obtain the scattering profiles as a function of $q$. The scattering profiles were corrected for the empty cell, electrical background and transmission. The sample-to-detector distance was $8 \mathrm{~m}$ for the measurement of the DPB-HPI and 4 and $12 \mathrm{~m}$ for the measurement of the microemulsion. The obtained profiles were normalized to the absolute unit $\left(\mathrm{cm}^{-1}\right)$ by using Lupolen ${ }^{\circledR}$ as a standard sample.

\section{Sample Preparation and Characterization of DPB-HPI}

Deuterated polybutadiene-block-polyisoprene diblock copolymer (DPB-HPI) was used in this work. The DPB-HPI was synthesized by sequential living anionic polymerization. The number-averaged molecular weight $M_{n}$ and polydispersity index $\left(M_{w} / M_{n}\right)$ of the DPB-HPI are $4.90 \times 10^{5}$ (GPC, equivalent to standard polystyrene) and 1.11 , respectively. Volume fraction of DPB is 0.53 . The microstructure of the DPB and the HPI was estimated from ${ }^{2} \mathrm{H}$ NMR and ${ }^{1} \mathrm{H}$ NMR measurements, respectively. The microstructure of the DPB is $94.8 \%$ 1,4 and $5.2 \% 1,2$ linkage. The microstructure of the HPI is $94.8 \% 1,4$ and $5.2 \% 3,4$ linkage.

\section{Results and Discussion for DPB-HPI}

In this section, we show the pressure dependencies of the order-disorder transition temperature $\left(T_{\text {ODT }}\right)$ and the microphase separation process induced by a pressure jump for the DPB-HPI block copolymer. A diblock copolymer has two different component polymer chains connected by a covalent bond. Therefore, even when there is strong net repulsion between the two components, only microphase separation occurs instead of macrophase separation as observed in the liquid mixtures of smallmolecules and polymer blends. ${ }^{12,13}$ The microphase separation occurs at a temperature below $T_{\text {ODT }}$ for the block copolymers of an upper critical order-disorder transition (ODT) temperature (UCODTT) type and above $T_{\text {ODT }}$ for the block copolymers of a lower critical ODT temperature (LCODTT) type: the spatial scale of the microphase is on the order of ten nanometers (i.e., the order of the radius of gyration of the polymer chain). DPB-HPI used in this work possesses an LCODTT-type microphase transition at least under atmospheric pressure $(P=0.1[\mathrm{MPa}])$. The details will be reported elsewhere. ${ }^{14}$ Recently, it has been pointed out that thermal noise effect strongly affects $T_{\mathrm{ODT}}$, giving a thermal fluctuation-induced first-order phase transition at $T_{\text {ODT }}{ }^{15-20}$ It is an intriguing physical problem to study how pressure affects the thermal noise and therefore $T_{\text {ODT }}$ and miscibility of block copolymers.

Pressure dependence of the scattering profiles for DPB-HPI at $341.4 \mathrm{~K}$ is shown in Figure 5. Figure 6 shows the magnified view of the scattering profiles around the second-order scattering peak in Figure 5 (at $q \cong 0.014$ $\left.\left[\AA^{-1}\right]\right)$. All the scattering profiles have the primary scattering maximum at $q_{\mathrm{m}} \cong 0.007\left[\AA^{-1}\right]$. Scattering profiles of diblock copolymers exhibit a broad scattering maximum even in the disordered state due to the correlation hole effect. ${ }^{15,21}$ On the other hand, the higher order peaks caused by a periodicity with long-range order are a strong indication of the ordered state. It is found from Figures 5 and 6 that the scattering profiles are significantly affected by magnitude of pressure applied. The maximum scattering intensity decreases with increasing pressure, indicating an increase in the miscibility. The second-order peak is observed at a pressure below $26[\mathrm{MPa}]$, indicating that DPB-HPI is in the ordered state, while the disappearance of the second-order peak at a pressure above $38[\mathrm{MPa}]$ suggests that it in the disordered state. This behavior suggests that DPB-HPI has a phase diagram (pressure-composition diagram) of an upper critical order-disorder transition pressure (UCODTP) type. Namely, DPB-HPI is in the disordered state under high pressure, and in the ordered state under low pressure. The pressure suppresses microphase separation and promotes miscibility of the block chains. It is predicted from the result in Figures 5 and 6 that the pressure at which the order-disorder transition occurs $\left(P_{\mathrm{ODT}}\right)$ is between 26 and $38[\mathrm{MPa}]$ at $T=341.4 \mathrm{~K}$. Meanwhile, the first- and second-order peaks are located at the scattering vectors in the ratio of $1: 2$, indicating that the alternating lamella structure is formed in the ordered state, which is consistent with the nearly symmetric composition of DPB-HPI.

Time-resolved neutron scattering measurements during the phase transition induced by a pressure jump were performed in order to investigate microphase separation process of DPB-HPI. Figure 7 shows the change in the scattering profiles with time (in seconds) after DPB- 


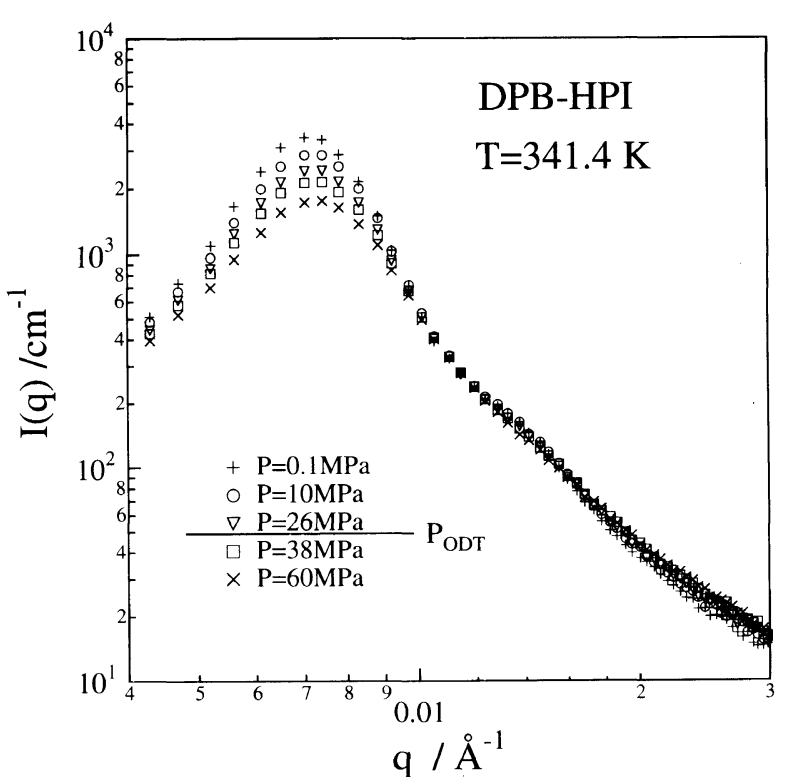

Figure 5. Scattering profiles for the DPB-HPI at various pressures at $T=341.4 \mathrm{~K}$.

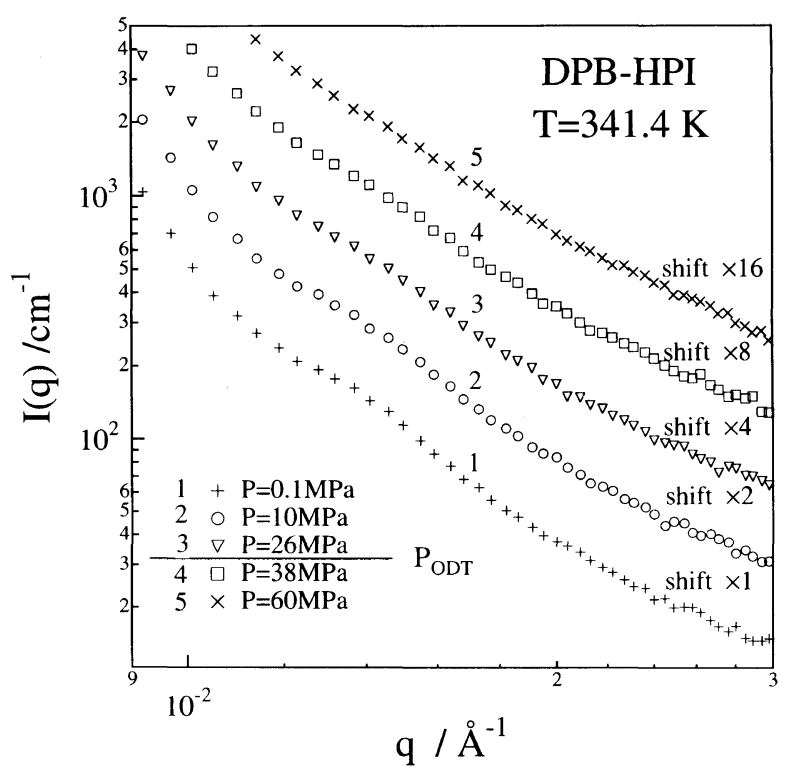

Figure 6. Magnification of Figure 6 around the second-order peak at $q \approx 0.014 \AA^{-1}$.

HPI was quenched from a disordered state to an ordered state by a pressure drop. Pressure was dropped from 80 (single-phase) to 0.1 [MPa] (microphase-separated) at $341.4 \mathrm{~K}$. Figure 8 shows a magnification of the scattering profiles around the second-order peak. Fluctuation of temperature was within $\pm 0.1 \mathrm{~K}$ during the measurements. The scattering profile in the equilibrium state before the pressure jump is also shown in Figures 7 and 8. To our surprise, at the moment of the pressure jump ( $t=0), q_{\mathrm{m}}$ (the value of $q$ at the scattering maximum) shifts toward lower $q$ as shown in Figure 7. We will present detailed analysis elsewhere. ${ }^{14}$ At later time $q_{\mathrm{m}}$ shifts back to its higher value. The scattered intensity also increases with time. Moreover, as shown in Figure 8 , the profiles at $t \geq 904.02 \mathrm{~s}$ start to exhibit a secondorder peak. The appearance of the second-order peak implies that ordering into lamella microdomains started

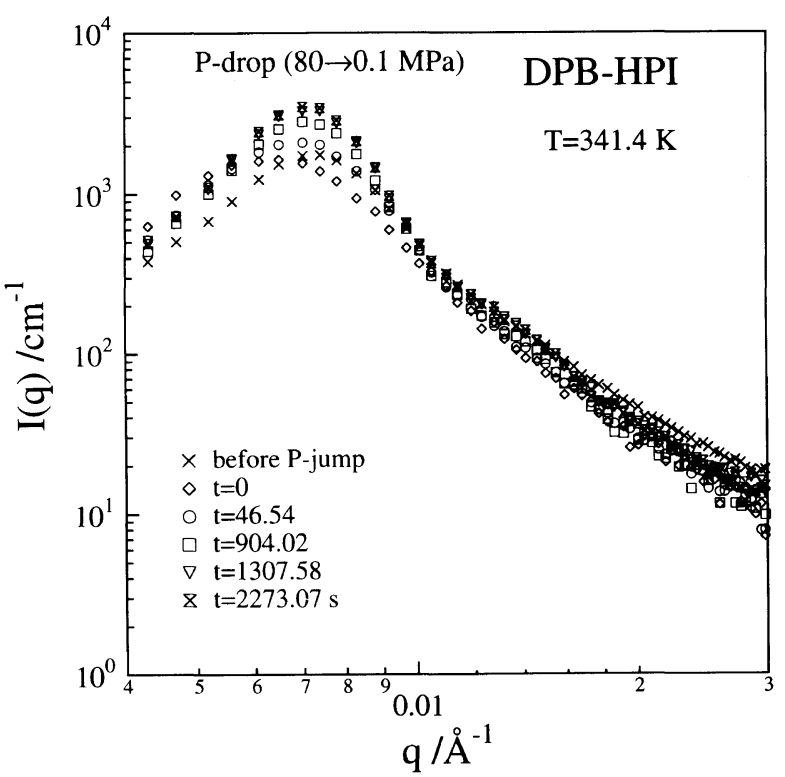

Figure 7. Time change in scattering profiles after the pressure drop (from $80 \mathrm{MPa}$ to $0.1 \mathrm{MPa}$ ) at $T=341.4 \mathrm{~K}$.

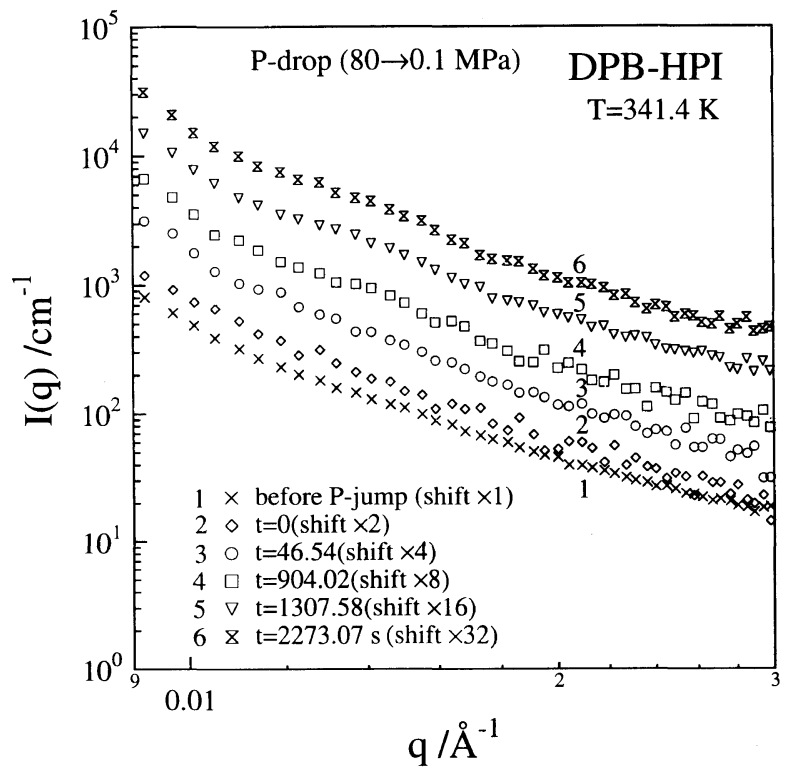

Figure 8. Magnification of Figure 8 around the second-order peak at $q \approx 0.014 \AA^{-1}$.

to occur with time. It was recently reported for a polystyrene-block-polyisoprene diblock copolymer that the microphase separation into lamella microdomains induced by temperature jump progresses via a nucleation and growth (NG) process with an incubation period of about $400 \mathrm{~s}$ at the quench depth $\Delta T$ of $2.7 \mathrm{~K}(\Delta T=$ $T_{\text {ODT }}-T$, where $T_{\text {ODT }}$ and $T$ are order-disorder transition temprerature and measuring temperature, respectively). ${ }^{22}$ The effect of pressure on the incubation time or the NG process will be reserved for future work. In addition, these detailed analyses including pressure dependencies of miscibility, $T_{\mathrm{ODT}}$, the thermal fluctuation effects and microphase separation process will be reported in the following paper. ${ }^{14}$

\section{Experiments on a Microemulsion System}

An oil-rich mixture of heavy water, $n$-decane, and AOT 
is a well-known system for investigating critical phenomena in a microemulsion system. At the oil-rich corner of the phase diagram, an uniform microemulsion is formed at room temperature and its structure is the socalled water-in-oil type, that is, water droplets coated by a surfactant film dispersed in oil pool. With increasing temperature, the system decomposes into two phases, a droplet-rich phase and a droplet-poor phase, and is associated with critical phenomena.

In previous studies, the system in the single-phase microemulsion system was thought to be a quasi-one component system, i.e., the droplet radius is constant through the phase separation and the droplet density $\phi$ can be treated as an order parameter. Thus, it is natural that these critical phenomena are explained within the framework of the 3D-Ising universality. Many experimentally observed critical exponents reveal values close to those of the 3D-Ising universality ${ }^{23,24}: \gamma=1.24$ and $v=0.63$, where $\gamma$ and $v$ are the exponents for the susceptibility $S_{0}$ of the droplet density fluctuations and for the correlation length $\xi$, respectively. Recent experimental results by Seto et al. ${ }^{25}$ indicate that the critical phenomena can be completely explained using the concept of the crossover from the mean-field to the 3D-Ising model. In this system, not only the temperature but also the hydrostatic pressure is a critical parameter. Kim et al. ${ }^{26}$ has shown an increase in the critical scattering with increasing pressure using LS and they conclude that the values of the critical exponents, $\gamma_{\mathrm{P}}=$ $1.5 \pm 0.05$ and $v_{\mathrm{P}}=0.7 \pm 0.03$ are consistent with those of the 3D-Ising model, where $\gamma_{\mathrm{P}}$ and $v_{\mathrm{P}}$ are defined as

$$
S_{0} \sim\left|P-P_{\mathrm{C}}\right|^{-\gamma_{\mathrm{P}}}, \quad \xi \sim\left|P-P_{\mathrm{C}}\right|^{-v_{\mathrm{P}}} .
$$

Eastoe et $a .^{27}$ have investigated the structures and properties of the similar microemulsion systems with varying temperature and pressure by means of SANS. They indicated that a change of the droplet radius with pressure is very small. This can be explained by a difference of compressibilities of ingredients; we believe their result is caused by the fact that the compressibility of water is smaller than that of normal alkane. This evidence indicates that the droplet density $\phi$, the volume fraction of the water and surfactant against the whole volumes, must increase with increasing pressure. In order to clarify the critical properties and structural change with applied pressure, we utilize the high pressure cell for SANS for the three-component microemulsion system.

AOT ( $99 \%$ purity), $n$-decane $(99 \%)$, and $\mathrm{D}_{2} \mathrm{O}(99.9 \%)$ were purchased from Fluka, Katayama Chemical, and Isotec Inc., respectively. They were used without purification and mixed within 5 days of opening the bottle of AOT to avoid a hydration. The water to surfactant molar ratio was fixed to be 40.86 following the previous results. $^{23-25}$ In this experiment, $\phi=0.098$ was selected because this composition is expected to lie very close to the critical point in the $T-\phi$ phase diagram. ${ }^{25}$

The measured pressure range was from 0.1 to 35.5 [MPa] with steps between 0.5 and $2.0[\mathrm{MPa}]$. The temperature was kept constant at $T=296.60 \mathrm{~K}$ with the accuracy of within $\pm 0.01 \mathrm{~K}$ during the measurements. Considering the change of the density of the components with increasing pressure, we calculate the density of heavy

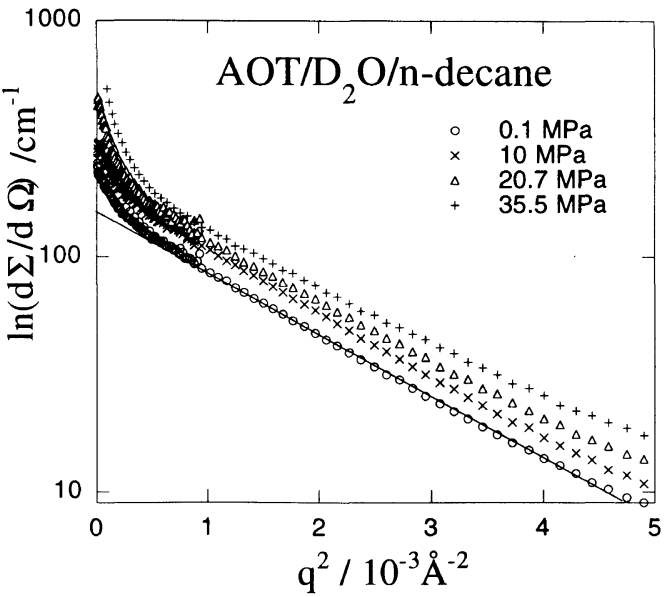

Figure 9. A Guinier plot of the scattering profiles at various pressures; the vertical axis indicates the logarithm of the scattering cross section and the horizontal axis is the square of the scattering vector $q$. The straight line indicates the fitted Guinier approximation.

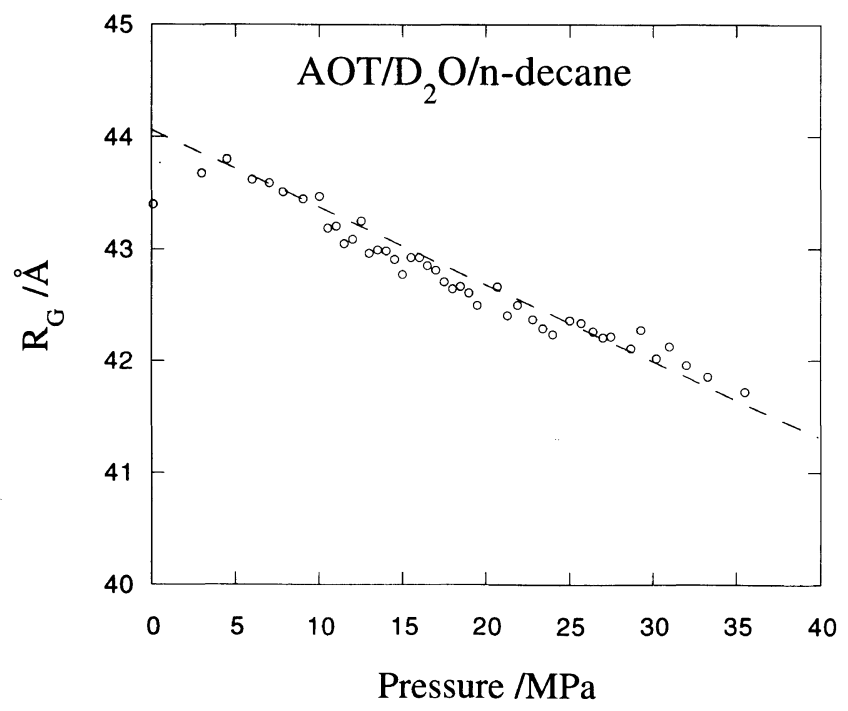

Figure 10. Pressure dependence of the observed Guinier radius $R_{\mathrm{G}}$. The dashed straight line is a guide to the eye.

water and $n$-decane from the compressibilities ${ }^{28,29}$ while that of AOT is treated as constant.

\section{Results and Discussions on a Microemulsion System}

Figure 9 shows the Guinier plot of scattering profiles at various pressures in the single phase state. The scattering intensity increased with increasing pressure, because the order parameter and the number density of droplets increases with pressure. (The scattering intensity was corrected for the thickness change with pressure, as discussed in section of INSTRUMENTATION. Correction for the Change of Sample Thickness) By fitting the Guinier approximation to the observed profiles over the range $2.64 \times 10^{-2}<q<5.32 \times 10^{-2}\left[\AA^{-1}\right]$, the Guinier radius $R_{\mathrm{G}}$ of droplets for various pressures is obtained. The pressure dependence of $R_{\mathrm{G}}$ is given in Figure 10. Upon approaching the critical point, the value of $R_{\mathrm{G}}$ decreases monotonically, contradicting the result of Eastoe et al. ${ }^{27}$ The tendency that $R_{\mathrm{G}}$ decreases on approaching the critical point is the same as the case of the temperature variation. $^{25}$ The decrease of the droplet radius might 


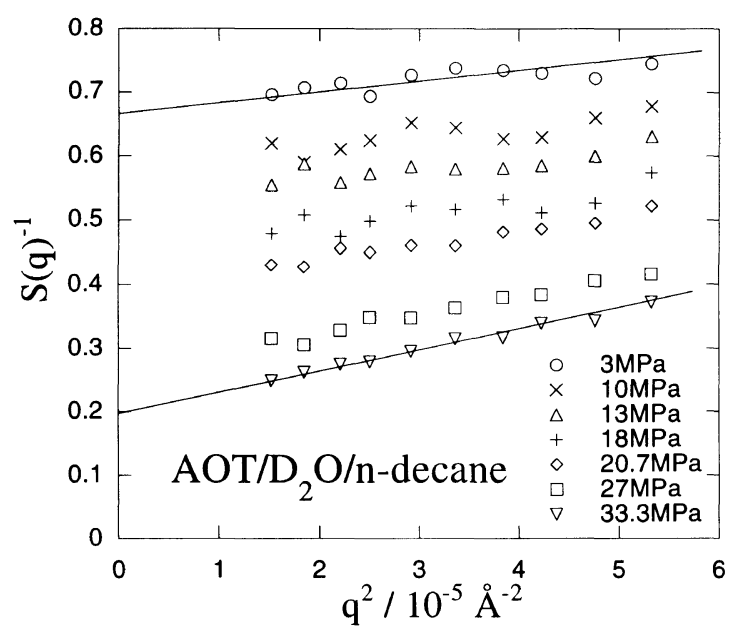

Figure 11. The scattering profiles at different pressures in the Zimm representation. Straight lines indicate the fitted Ornstein-Zernike formula.

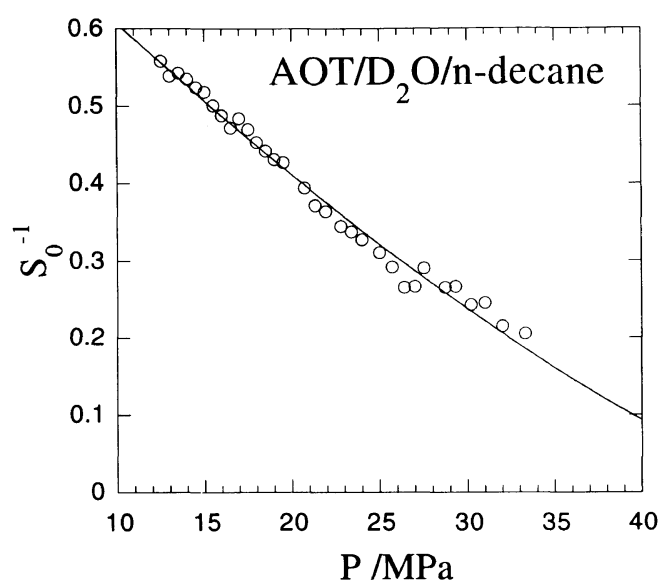

Figure 12. Pressure variations of the observed inverse forward scattering. The line indicates fitting curve for a critical phenomenon expressed in the eq 3.

result from the change of the hydrophile-lypophile balance with increasing pressure. In the low- $q$ region $\left(q<7.4 \times 10^{-3}\left[\AA^{-1}\right]\right)$, the structure factor $S(q)$ follows the Ornstein-Zernike approximation. Figure 11 shows the inverse of $S(q)$ against $q^{2}$ for various pressures. With increasing pressure, the inverse forward scattering $S_{0}^{-1}$ tends to zero. In Figure 12, the observed $S_{0}^{-1}$ is given as a function of pressure $P$. The line is a fit to the formula

$$
S_{0}^{-1} \sim\left|P-P_{\mathrm{C}}\right|^{\gamma_{\mathrm{P}}}
$$

where $P_{\mathrm{C}}$ is the critical pressure. The critical exponent $\gamma_{\mathrm{P}}=1.4 \pm 0.3$ and the critical pressure $P_{\mathrm{C}}=50.1 \pm 6.0$ $[\mathrm{MPa}]$ are obtained. This value of $\gamma_{\mathrm{P}}$ is consistent with the value observed by Kim et al. ${ }^{26}$ We believe that the results of $\mathrm{LS}^{26}$ are confirmed by this SANS experiment. This system shows the lower critical solution pressure (LCSP) type phase diagram in contrast to the polymer systems studied in section of EXPERIMENTAL APPLICATION: Results and Discussion for DPB-HPI.

\section{SUMMARY}

We have designed and constructed high pressure cells for both small-angle neutron scattering (SANS) and light scattering (LS) studies in order to investigate pressure effects on phase transitions of complex liquids. We can investigate structures of a wide spatial scale by using the cells. The cells can be used for both high and low viscosity liquids. Moreover, dynamics of phase transitions induced by a pressure drop can be measured by using the cells. The maximum pressure that can be applied to the cells is $c a .200$ [MPa], and the stability of pressure control is within $\pm 0.5[\mathrm{MPa}]$ during the measurement time of several hours. Preliminary experiments on phase transitions of diblock copolymer and microemulsion systems which are high and low viscosity liquids, respectively, have been carried out using the cell for SANS studies. While the former has a phase diagram of UCODTP type, the latter has a LCSP-type phase diagram. Their phase transitions are significantly affected by the applied pressure.

Acknowledgments. The authors are grateful to $\mathrm{Mr}$. M. Koizumi, Hikari High-pressure Machinery Co., Ltd., for the construction of the high-pressure cells and for Dr. Yoneyama, Asahi Chemical Industry Co., Ltd., for helping with the design. The authors thank Dr. K. Tsutsumi, Hashimoto Polymer Phasing Project, ERATO, JST, Japan for helping with NMR measurements. The authors are grateful to Professor S. Komura at Hiroshima University, Japan for valuable discussion. This work was supported in part by a "Grant-in-Aid for Encouragement of Young Scientists of the Ministry of Education, Science, and Culture" (No. 07740329) for the Hiroshima University group and by that on Priority Areas, "Cooperative Phenomena in Complex Liquids" (07236103) for the Kyoto University group, both from the Ministry of Education, Science, Sports and Culture, Japan (07651106). This work was performed with the approval of the Neutron Scattering Program Advisory Committee (Proposal No. 95-25 for the Kyoto University group and No. 95-77 for the Hiroshima University group).

\section{REFERENCES}

1. T. Hashimoto, Phase Transitions, 12, 47 (1988).

2. K. Binder, Ad. Polym. Sci., 112, 181 (1994).

3. P. J. Flory, J. Am. Chem. Soc., 87, 1833 (1965).

4. I. C. Sanchez and R. H. Lacombe, J. Phys. Chem., 80, 2352 (1976).

5. S. Janssen, D. Schwahn, K. Mortensen, and T. Springer, Macromolecules, 26, 5587 (1993).

6. H. Frielinghaus, "SANS-Untersuchungen der Druckabhängigkeit von Diblockcopolymer-Phasendiagrammen," Diploma thesis, Rheinisch-Westfälische Technische Hochshule Aachen, 1994.

7. B. Hammouda and B. J. Bauer, Macromolecules, 28, 4505 (1995).

8. D. A. Hajduk, P. Urayama, M. Gruner, S. Erramilli, R. A. Register, K. Brister, and L. J. Fetters, Macromolecules, 28, 7148 (1995).

9. J. Kojima, M. Takenaka, Y. Nakayama, and T. Hashimoto, Rev. Sci. Instrum., 66, 4066 (1995).

10. P. J. Flory, "Principles of Polymer Chemistry," Cornell University Press, Ithaca, N. Y., 1953.

11. B. Rudolf and H. Cantow, Macromolecules, 28, 6586 (1995).

12. T. Hashimoto, in "Thermoplastic Elastomers," 2nd ed, N. R. Legge, G. Holden, and H. E. Schroeder, Ed., Hanser, Vienna, 1987, Chapter 12, Section 3, 1996, p 349.

13. F. S. Bates and G. H. Fredrickson, Annu. Rev. Phys. Chem., 41, 525 (1990).

14. H. Takeno, H. Hasegawa, N. Sakamoto, T. Hashimoto, and M. Imai, Macromolecules, in preparation.

Polym. J., Vol. 29, No. 11, 1997 
15. L. Leibler, Macromolecules, 13, 1602 (1980).

16. G. H. Fredrickson and E. Helfand, J. Chem. Phys., 87, 697 (1987).

17. A. Brazovskii, A. Sov. Phys.-JETP (Engl. Transl.), 41, 85 (1975)

18. F. S. Bates, J. H. Rosedale, and G. H. Fredrickson, J. Chem. Phys., 92, 6255 (1990).

19. T. Hashimoto, T. Ogawa, and C. D. Han, J. Phys. Soc. Jpn., 63, 2206 (1994).

20. N. Sakamoto and T. Hashimoto, Macromolecules, 28, 6825 (1995).

21. P. G. de Gennes, "Scaling Concepts in Polymer Physics," Cornell University, Ithaca, NY, 1979.

22. T. Hashimoto and N. Sakamoto, Macromolecules, 28, 4779 (1995).
23. M. Kotlarchyk, S. H. Chen, J. S. Huang, and M. W. Kim, Phys. Rev. A, 29, 2054 (1983).

24. J. Rouch, A. Safouane, P. Tartaglia, and S. H. Chen, J. Chem. Phys., 90, 3756 (1989).

25. H. Seto, D. Schwahn, M. Nagao, E. Yokoi, S. Komura, M. Imai, and K. Mortensen, Phys. Rev. E, 54, 629 (1996).

26. M. W. Kim, J. Bock, and J. S. Huang, Phys. Rev. Lett., 54, 46 (1984).

27. J. Eastoe, D. C. Steytler, B. H. Robinson, and R. K. Heenan, J. Chem. Soc., Faraday Trans., 90, 3121 (1994).

28. P. W. Bridgeman, J. Chem. Phys., 3, 597 (1936).

29. S. Toxvaerd, J. Chem. Phys., 93, 4290 (1990). 